CUBO A Mathematical Journal

Vol.12, $N^{\underline{O}} 01$, (23-40). March 2010

\title{
Quenching for Discretizations of a Nonlocal Parabolic Problem with Neumann Boundary Condition
}

\author{
THÉODORE K. BONI \\ Institut National Polytechnique Houphouët-Boigny de Yamoussoukro, \\ BP 1093 Yamoussoukro, (Côte d'Ivoire) \\ email: theokboni@yahoo.fr \\ AND \\ DiABAté NABONGO \\ Université d'Abobo-Adjamé, UFR-SFA, \\ Département de Mathématiques et Informatiques, \\ 16 BP 372 Abidjan 16, (Côte d'Ivoire) \\ email: nabongo_diabate@yahoo.fr
}

\begin{abstract}
In this paper, under some conditions, we show that the solution of a discrete form of a nonlocal parabolic problem quenches in a finite time and estimate its numerical quenching time. We also prove that the numerical quenching time converges to the real one when the mesh size goes to zero. Finally, we give some computational results to illustrate our analysis.
\end{abstract}

\section{RESUMEN}

En este artículo mostramos, bajo algunas condiciones, que la solución de una forma discreta de un problema parabólico no local se sofoca en tiempo finito y estimamos su tiempo de sofocamiento numérico. Probamos también que el tiempo de sofocamiento numérico converge par un real cuando el tamaño de la malla tiende a cero. Finalmente damos algunos resultados computacionales para ilustrar nuestros análisis. 
Key words and phrases: Nonlocal diffusion, quenching, numerical quenching time.

Math. Subj. Class.: 5B40, 45A07, 45G10, 65M06.

\section{Introduction}

Consider the following initial value problem

$$
\begin{gathered}
u_{t}=\int_{\Omega} J(x-y)(u(y, t)-u(x, t)) d y+(M-u)^{-p} \quad \text { in } \bar{\Omega} \times(0, T), \\
u(x, 0)=u_{0}(x) \geq 0 \text { in } \bar{\Omega},
\end{gathered}
$$

where $\Omega=(-1,1)^{N}, M=$ const $>0, p=$ const $>0, J: \mathbb{R}^{N} \rightarrow \mathbb{R}$ is a $C^{1}$ nonnegative function. In addition, $J$ is symmetric $(J(z)=J(-z))$, and $\int_{\mathbb{R}^{N}} J(z) d z=1$. The initial data $u_{0} \in C^{0}(\bar{\Omega})$, $0 \leq u_{0}(x)<M, x \in \bar{\Omega}$.

Here, $(0, T)$ is the maximal time interval on which the solution $u$ exists. The time $T$ may be finite or infinite. When $T$ is infinite, then we say that the solution $u$ exists globally. When $T$ is finite, then the solution $u$ develops a singularity in a finite time, namely,

$$
\lim _{t \rightarrow T}\|u(\cdot, t)\|_{\infty}=M
$$

where $\|u(\cdot, t)\|_{\infty}=\sup _{x \in \bar{\Omega}}|u(x, t)|$. In this last case, we say that the solution $u$ quenches in a finite time, and the time $T$ is called the quenching time of the solution $u$. Recently, nonlocal diffusion has been the subject of investigation of many authors (see, [1]-[7], [10]-[12], [14]-[18], [20], [24], [27], and the references cited therein). Nonlocal evolution equations of the form

$$
u_{t}=\int_{\mathbb{R}^{N}} J(x-y)(u(y, t)-u(x, t)) d y,
$$

and variations of it, have been used by several authors to model diffusion processes (see, [3], [4], [10], [17], [18]). The solution $u(x, t)$ can be interpreted as the density of a single population at the point $x$, at the time $t$, and $J(x-y)$ as the probability distribution of jumping from location $y$ to location $x$. Then, the convolution $(J * u)(x, t)=\int_{\mathbb{R}^{N}} J(x-y) u(y, t) d y$ is the rate at which individuals are arriving to position $x$ from all other places, and $-u(x, t)=-\int_{\mathbb{R}^{N}} J(x-y) u(x, t) d y$ is the rate at which they are leaving location $x$ to travel to any other site (see, [17]). Let us notice that the reaction term $(M-u)^{-p}$ in the equation (1) can be rewritten as follows

$$
(M-u(x, t))^{-p}=\int_{\mathbb{R}^{N}} J(x-y)(M-u(x, t))^{-p} d y .
$$

Therefore, in view of the above equality, the reaction term $(M-u)^{-p}$ can be interpreted as a force that decreases the rate at which individuals are leaving location $x$ to travel to any other site. Due to the presence of the term $(M-u)^{-p}$, we shall see later that the phenomenon of quenching occurs 
for the density $u(x, t)$. On the other hand, the integral in (1) is taken over $\Omega$. Thus, there is no individuals that enter or leave the domain $\Omega$. It is the reason why in the title of the paper, we have added Neumann boundary condition. In the current paper, we are interested in the numerical study of the phenomenon of quenching using a discrete form of (1)-(2). Let us notice that, setting $v=M-u$, the problem (1)-(2) is equivalent to

$$
\begin{gathered}
v_{t}(x, t)=\int_{\Omega} J(x-y)(v(y, t)-v(x, t)) d y-v^{-p} \quad \text { in } \quad \bar{\Omega} \times(0, T), \\
v(x, 0)=v_{0}(x)>0 \quad \text { in } \bar{\Omega},
\end{gathered}
$$

where $v_{0}(x)=M-u_{0}(x)$. Consequently, the solution $u$ of (1)-(2) quenches at the time $T$ if and only if the solution $v$ of (3)-(4) quenches at the time $T$, that is,

$$
\lim _{t \rightarrow T} v_{\min }(t)=0
$$

where $v_{\min }(t)=\min _{\bar{\Omega}} v(x, t)$. Thus, by convenience, we shall often utilize the problem (3)-(4) instead of (1)-(2). We start by the construction of an explicit adaptive scheme as follows. Let $I$ be a positive integer, and let $h=2 / I$. Define the grid $x_{i}=-1+i h, 0 \leq i \leq I$, and approximate the solution $v$ of (3)-(4) by the solution $U_{h}^{(n)}=\left(U_{K}^{(n)}\right)_{K \in \Gamma}$ of the following discrete equations

$$
\begin{gathered}
\delta_{t} U_{K}^{(n)}=\sum_{L \in \Gamma_{*}} h^{N} J\left(x_{K}-x_{L}\right)\left(U_{L}^{(n)}-U_{K}^{(n)}\right)-\left(U_{K}^{(n)}\right)^{-p}, K \in \Gamma, n \geq 0, \\
U_{K}^{(0)}=\varphi_{K}, \quad K \in \Gamma,
\end{gathered}
$$

where

$$
\begin{gathered}
\Gamma=\left\{\left(j_{1}, \cdots, j_{N}\right) ; 0 \leq j_{1}, \cdots, j_{N} \leq I\right\}, \\
\Gamma_{*}=\left\{\left(j_{1}, \cdots, j_{N}\right) ; \quad 0 \leq j_{1}, \cdots, j_{N} \leq I-1\right\}, \\
x_{K}=\left(x_{k_{1}}, \cdots, x_{k_{N}}\right), \quad x_{L}=\left(x_{l_{1}}, \cdots, x_{l_{N}}\right),
\end{gathered}
$$

and

$$
\delta_{t} U_{K}^{(n)}=\frac{U_{K}^{(n+1)}-U_{K}^{(n)}}{\Delta t_{n}} .
$$

In order to permit the discrete solution to reproduce the properties of the continuous one when the time $t$ approaches the quenching time $T$, we need to adapt the size of the time step so that we take

$$
\Delta t_{n}=\min \left\{h^{2}, \tau\left(U_{h \min }^{(n)}\right)^{p+1}\right\},
$$

where $U_{h m i n}^{(n)}=\min _{K \in \Gamma} U_{K}^{(n)}, \tau \in(0,1)$. Let us notice that the restriction on the time step ensures the positivity of the discrete solution.

To facilitate our discussion, we need to define the notion of numerical quenching time. 
Definition 1.1. We say that the discrete solution $U_{h}^{(n)}$ of (5)-(6) quenches in a finite time if $\lim _{n \rightarrow \infty} U_{h m i n}^{(n)}=0$, and the series $\sum_{n=0}^{\infty} \Delta t_{n}$ converges. The quantity $\sum_{n=0}^{\infty} \Delta t_{n}$ is called the numerical quenching time of the discrete solution $U_{h}^{(n)}$.

In the present paper, under some conditions, we show that the discrete solution quenches in a finite time and estimate its numerical quenching time. We also show that the numerical quenching time converges to the real one when the mesh size goes to zero. A similar result has been obtained by Nabongo and Boni in [25] within the framework of the phenomenon of quenching for local parabolic problems. One may also consult the papers of the same authors in [22] and [23] for numerical studies of the phenomenon of quenching where semidiscretizations in space have been utilized. The remainder of the paper is organized as follows. In the next section, we reveal certain properties of the continuous problem. In the third section, we exhibit some features of the discrete scheme. In the fourth section, under some assumptions, we demonstrate that the discrete solution quenches in a finite time, and estimate its numerical quenching time. In the fifth section, the convergence of the numerical quenching time is analyzed, and finally, in the last section, we show some numerical experiments to illustrate our analysis.

\section{Local Existence}

In this section, we shall establish the existence and uniqueness of solutions of (1)-(2) in $\Omega \times(0, T)$ for all small $T$. Some results about quenching are also given.

Let $t_{0}>0$ be fixed, and define the function space $Y_{t_{0}}=\left\{u ; u \in C\left(\left[0, t_{0}\right], C(\bar{\Omega})\right)\right\}$ equipped with the norm defined by $\|u\|_{Y_{t_{0}}}=\max _{0 \leq t \leq t_{0}}\|u(\cdot, t)\|_{\infty}$ for $u \in Y_{t_{0}}$. It is easy to see that $Y_{t_{0}}$ is a Banach space. Introduce the set

$$
X_{t_{0}}=\left\{u ; u \in Y_{t_{0}},\|u\|_{Y_{t_{0}}} \leq b_{0}\right\},
$$

where $b_{0}=\frac{\left\|u_{0}\right\|_{\infty}+M}{2}$. We observe that $X_{t_{0}}$ is a nonempty bounded closed convex subset of $Y_{t_{0}}$. Define the map $R$ as follows

$$
\begin{gathered}
R: X_{t_{0}} \rightarrow X_{t_{0}} \\
R(v)(x, t)=u_{0}(x)+\int_{0}^{t} \int_{\Omega} J(x-y)(v(y, s)-v(x, s)) d y d s+\int_{0}^{t}(M-v(x, s))^{-p} d s .
\end{gathered}
$$

Theorem 2.1. Assume that $u_{0} \in C(\bar{\Omega})$. Then $R$ maps $X_{t_{0}}$ into $X_{t_{0}}$, and $R$ is strictly contractive if $t_{0}$ is appropriately small relative to $\left\|u_{0}\right\|_{\infty}$.

Proof. Due to the fact that $\int_{\Omega} J(x-y) d y \leq \int_{\mathbb{R}^{N}} J(x-y) d y=1$, a straightforward computation reveals that

$$
\left|R(v)(x, t)-u_{0}(x)\right| \leq 2\|v\|_{Y_{t_{0}}} t+\left(M-\|v\|_{Y_{t_{0}}}\right)^{-p} t,
$$

which implies that $\|R(v)\|_{Y_{t_{0}}} \leq\left\|u_{0}\right\|_{\infty}+2 b_{0} t_{0}+\left(M-b_{0}\right)^{-p} t_{0}$. If

$$
t_{0} \leq \frac{b_{0}-\left\|u_{0}\right\|_{\infty}}{2 b_{0}+\left(M-b_{0}\right)^{-p}}
$$


then $\|R(v)\|_{Y_{t_{0}}} \leq b_{0}$. Therefore, if (7) holds, then $R$ maps $X_{t_{0}}$ into $X_{t_{0}}$. Now, we are going to prove that the map $R$ is strictly contractive. Letting $v, z \in X_{t_{0}}$ and setting $\alpha=v-z$, we discover that

$$
\begin{aligned}
|(R(v)-R(z))(x, t)| & \leq\left|\int_{0}^{t} \int_{\Omega} J(x-y)(\alpha(y, s)-\alpha(x, s)) d y d s\right| \\
& +\left|\int_{0}^{t}\left((M-v(x, s))^{-p}-(M-z(x, s))^{-p}\right) d s\right| .
\end{aligned}
$$

Use Taylor's expansion to obtain

$$
|(R(v)-R(z))(x, t)| \leq 2\|\alpha\|_{Y_{t_{0}}} t+t\|v-z\|_{Y_{t_{0}}} p\left(M-\|\beta\|_{Y_{t_{0}}}\right)^{-p-1},
$$

where $\beta$ is a function which is localized between $v$ and $z$. We deduce that

$$
\|R(v)-R(z)\|_{Y_{t_{0}}} \leq 2\|\alpha\|_{Y_{t_{0}}} t_{0}+t_{0}\|v-z\|_{Y_{t_{0}}} p\left(M-\|\beta\|_{Y_{t_{0}}}\right)^{-p-1},
$$

which implies that $\|R(v)-R(z)\|_{Y_{t_{0}}} \leq\left(2 t_{0}+t_{0} p\left(M-b_{0}\right)^{-p-1}\right)\|v-z\|_{Y_{t_{0}}}$. If

$$
t_{0} \leq \frac{1}{4+2 p\left(M-b_{0}\right)^{-p-1}},
$$

then $\|R(v)-R(z)\|_{Y_{t_{0}}} \leq \frac{1}{2}\|v-z\|_{Y_{t_{0}}}$. Hence, we see that $R(v)$ is a strict contraction in $Y_{t_{0}}$, and the proof is complete.

It follows from the contraction mapping principle that for appropriately chosen $t_{0}, R$ has a unique fixed point $u \in Y_{t_{0}}$ which is a solution of (1)-(2). If $\|u\|_{Y_{t_{0}}}<M$, then taking as initial data $u\left(\cdot, t_{0}\right) \in C(\bar{\Omega})$ and arguing as before, it is possible to extend the solution up to some interval $\left[0, t_{1}\right)$ for certain $t_{1}>t_{0}$. Hence, we conclude that if the maximal time interval of existence of the solution, $(0, T)$, is finite then the solution quenches in a finite time in $L^{\infty}(\bar{\Omega})$ norm, namely, $\lim _{t \rightarrow T}\|u(\cdot, t)\|_{\infty}=M$.

Remark 2.1. Let us notice that we can define the map $R$ in the space $Y_{t_{0}}=C^{1,2}\left(\bar{\Omega} \times\left[0, t_{0}\right]\right)$. Besides, if $u_{0} \in C^{1}(\bar{\Omega})$, then arguing as in the proof of Theorem 2.1, it is not hard to see that Theorem 2.1 remains valid. Consequently, if $u_{0} \in C^{1}(\bar{\Omega})$, then the solution $u$ of (1)-(2) belongs to $C^{1,2}(\bar{\Omega} \times[0, T))$ when $T$ is finite.

The following lemma is a version of the maximum principle for nonlocal problems.

Lemma 2.1. Let $a \in C^{0}(\bar{\Omega} \times[0, T))$, and let $u \in C^{0,1}(\bar{\Omega} \times[0, T))$ satisfy the following inequalities

$$
\begin{gathered}
u_{t}-\int_{\Omega} J(x-y)(u(y, t)-u(x, t)) d y+a(x, t) u(x, t) \geq 0 \quad \text { in } \bar{\Omega} \times(0, T), \\
u(x, 0) \geq 0 \quad \text { in } \bar{\Omega} .
\end{gathered}
$$

Then, we have $u(x, t) \geq 0$ in $\bar{\Omega} \times(0, T)$. 
Proof. Let $T_{0}$ be any positive quantity satisfying $T_{0}<T$. Since $a(x, t)$ is bounded in $\bar{\Omega} \times\left[0, T_{0}\right]$, then there exists $\lambda$ such that $a(x, t)-\lambda>0$ in $\bar{\Omega} \times\left[0, T_{0}\right]$. Define $z(x, t)=e^{\lambda t} u(x, t)$ and let $m=\min _{x \in \bar{\Omega}, t \in\left[0, T_{0}\right]} z(x, t)$. Due to the fact that $z$ is continuous in $\bar{\Omega} \times\left[0, T_{0}\right]$, then it achieves its minimum in $\bar{\Omega} \times\left[0, T_{0}\right]$. Consequently, there exists $\left(x_{0}, t_{0}\right) \in \bar{\Omega} \times\left[0, T_{0}\right]$ such that $m=z\left(x_{0}, t_{0}\right)$. We get $z\left(x_{0}, t_{0}\right) \leq z\left(x_{0}, t\right)$ for $t \leq t_{0}$ and $z\left(x_{0}, t_{0}\right) \leq z\left(y, t_{0}\right)$ for $y \in \Omega$. This implies that

$$
z_{t}\left(x_{0}, t_{0}\right) \leq 0, \quad \int_{\Omega} J\left(x_{0}-y\right)\left(z\left(y, t_{0}\right)-z\left(x_{0}, t_{0}\right)\right) d y \geq 0 .
$$

With the aid of the first inequality of the lemma, it is not hard to see that

$$
z_{t}\left(x_{0}, t_{0}\right)-\int_{\Omega} J\left(x_{0}-y\right)\left(z\left(y, t_{0}\right)-z\left(x_{0}, t_{0}\right)\right) d y+\left(a\left(x_{0}, t_{0}\right)-\lambda\right) z\left(x_{0}, t_{0}\right) \geq 0 .
$$

We deduce from (11) that $\left(a\left(x_{0}, t_{0}\right)-\lambda\right) z\left(x_{0}, t_{0}\right) \geq 0$. Since $a\left(x_{0}, t_{0}\right)-\lambda>0$, we get $z\left(x_{0}, t_{0}\right) \geq 0$. This implies that $u(x, t) \geq 0$ in $\bar{\Omega} \times\left[0, T_{0}\right]$, and the proof is complete.

An immediate consequence of the above lemma is that the solution $u$ of (1)-(2) is nonnegative in $\bar{\Omega} \times(0, T)$ because the initial data $u_{0}(x)$ is nonnegative in $\bar{\Omega}$.

Now, let us give a result about quenching which says that the solution $u$ of (1)-(2) always quenches in a finite time. This assertion is stated in the theorem below.

Theorem 2.2. The solution $u$ of (1)-(2) quenches in a finite time, and its quenching time $T$ satisfies the following estimate

$$
T \leq \frac{(M-A)^{p+1}}{p+1},
$$

where $A=\frac{1}{|\Omega|} \int_{\Omega} u_{0}(x) d x$.

Proof. Since $(0, T)$ is the maximal time interval of existence of the solution $u$, our aim is to show that $T$ is finite and satisfies the above inequality. Due to the fact that the initial data $u_{0}(x)$ is nonnegative in $\bar{\Omega}$, we know from Lemma 2.1 that the solution $u(x, t)$ of (1)-(2) is nonnegative in $\bar{\Omega} \times(0, T)$. Integrating both sides of $(1)$ over $(0, t)$, we find that

$$
\begin{aligned}
u(x, t)-u_{0}(x) & =\int_{0}^{t} \int_{\Omega} J(x-y)(u(y, s)-u(x, s)) d y d s \\
& +\int_{0}^{t}(M-u(x, s))^{-p} d s \quad \text { for } \quad t \in(0, T) .
\end{aligned}
$$

Integrate again in the $x$ variable and apply Fubini's theorem to obtain

$$
\int_{\Omega} u(x, t) d x-\int_{\Omega} u_{0}(x) d x=\int_{0}^{t}\left(\int_{\Omega}(M-u(x, s))^{-p} d x\right) d s \text { for } t \in(0, T) .
$$

Set

$$
w(t)=\frac{1}{|\Omega|} \int_{\Omega} u(x, t) d x \quad \text { for } \quad t \in[0, T) .
$$


Taking the derivative of $w$ in $t$ and using (13), we arrive at

$$
w^{\prime}(t)=\int_{\Omega} \frac{1}{|\Omega|}(M-u(x, t))^{-p} d x \quad \text { for } \quad t \in(0, T) .
$$

It follows from Jensen's inequality that $w^{\prime}(t) \geq(M-w(t))^{-p}$ for $t \in(0, T)$, or equivalently

$$
(M-w)^{p} d w \geq d t \quad \text { for } \quad t \in(0, T) .
$$

Integrate the above inequality over $(0, T)$ to obtain

$$
T \leq \frac{(M-w(0))^{p+1}}{p+1} .
$$

Since the quantity on the right hand side of the above inequality is finite, we deduce that $u$ quenches in a finite time at the time $T$ which obeys the above inequality. Use the fact that $w(0)=A$ to complete the rest of the proof.

\section{Properties of the Semidiscrete Scheme}

In this section, we give some results about the discrete maximum principle of nonlocal problems for our subsequent use.

The lemma below is a discrete version of the maximum principle for nonlocal parabolic problems.

Lemma 3.1. For $n \geq 0$, let $U_{h}^{(n)}, a_{h}^{(n)}$ be two vectors such that

$$
\begin{gathered}
\delta_{t} U_{K}^{(n)} \geq \sum_{L \in \Gamma_{*}} h^{N} J\left(x_{K}-x_{L}\right)\left(U_{L}^{(n)}-U_{K}^{(n)}\right)+a_{K}^{(n)} U_{K}^{(n)}, K \in \Gamma, n \geq 0, \\
U_{K}^{(0)} \geq 0, \quad K \in \Gamma .
\end{gathered}
$$

Then, we have $U_{K}^{(n)} \geq 0, K \in \Gamma, n>0$ when $\Delta t_{n} \leq \frac{1}{2^{N}\|J\|_{\infty}+\left\|a_{h}^{(n)}\right\|_{\infty}}$, where $\left\|a_{h}^{(n)}\right\|_{\infty}=$ $\sup _{K \in \Gamma}\left|a_{K}^{(n)}\right|$.

Proof. If $U_{h}^{(n)} \geq 0$, then a straightforward computation reveals that

$$
U_{K}^{(n+1)} \geq U_{K}^{(n)}\left(1-2^{N}\|J\|_{\infty} \Delta t_{n}-\left\|a_{h}^{(n)}\right\|_{\infty} \Delta t_{n}\right), \quad K \in \Gamma, \quad n \geq 0 .
$$

To obtain the above inequalities, we have used the fact that

$$
\sum_{L \in \Gamma_{*}} h^{N} J\left(x_{K}-x_{L}\right) \geq 0, \quad K \in \Gamma,
$$

and

$$
\sum_{L \in \Gamma_{*}} h^{N} J\left(x_{K}-x_{L}\right) \leq\|J\|_{\infty}\left(\sum_{l=0}^{I-1} h\right)^{N}=2^{N}\|J\|_{\infty}, \quad K \in \Gamma .
$$


Making use of (15) and an argument of recursion, we easily check that $U_{h}^{(n+1)} \geq 0, n \geq 0$. This finishes the proof.

An immediate consequence of the above result is the following comparison lemma. Its proof is straightforward.

Lemma 3.2. For $n \geq 0$, let $U_{h}^{(n)}, V_{h}^{(n)}$ and $a_{h}^{(n)}$ be three vectors such that

$$
\begin{gathered}
\delta_{t} U_{K}^{(n)}-\sum_{L \in \Gamma_{*}} h^{N} J\left(x_{K}-x_{L}\right)\left(U_{L}^{(n)}-U_{K}^{(n)}\right)+a_{K}^{(n)} U_{K}^{(n)} \\
\geq \delta_{t} V_{K}^{(n)}-\sum_{L \in \Gamma_{*}} h^{N} J\left(x_{K}-x_{L}\right)\left(V_{L}^{(n)}-V_{K}^{(n)}\right)+a_{K}^{(n)} V_{K}^{(n)}, \quad K \in \Gamma, \quad n \geq 0, \\
U_{K}^{(0)} \geq V_{K}^{(0)}, \quad K \in \Gamma .
\end{gathered}
$$

Then, we have $U_{K}^{(n)} \geq V_{K}^{(n)}, K \in \Gamma, n>0$ when $\Delta t_{n} \leq \frac{1}{2^{N}\|J\|_{\infty}+\left\|a_{h}^{(n)}\right\|_{\infty}}$.

Remark 3.1. For $n \geq 0$, introduce the vector $Z_{h}^{(n)}$ defined as follows $Z_{K}^{(n)}=\left\|\varphi_{h}\right\|_{\infty}-U_{K}^{(n)}$, $K \in \Gamma$, where $U_{h}^{(n)}$ is the solution of (5)-(6). A straightforward computation reveals that

$$
\begin{gathered}
\delta_{t} Z_{K}^{(n)} \geq \sum_{L \in \Gamma_{*}} h^{N} J\left(x_{K}-x_{L}\right)\left(Z_{L}^{(n)}-Z_{K}^{(n)}\right), \quad K \in \Gamma, \quad n \geq 0, \\
Z_{K}^{(0)} \geq 0, \quad K \in \Gamma .
\end{gathered}
$$

It follows from Lemma 3.1 that $\left\|\varphi_{h}\right\|_{\infty} \geq U_{K}^{(n)}, K \in \Gamma, n>0$ when $h$ is small enough.

\section{The Numerical Quenching Time}

In this section, under some assumptions, we show that the discrete solution quenches in a finite time and estimate its numerical quenching time.

Our result concerning the numerical quenching time is stated in the following theorem.

Theorem 4.1. Assume that the initial data at (6) satisfies $2^{N}\|J\|_{\infty}\left\|\varphi_{h}\right\|_{\infty}^{p+1}<1$. Then, the discrete solution $U_{h}^{(n)}$ of (5)-(6) quenches in a finite time, and its quenching time $T_{h}^{\Delta t}$ obeys the following estimate

$$
T_{h}^{\Delta t} \leq \frac{\tau \varphi_{h m i n}^{p+1}}{1-\left(1-\tau^{\prime}\right)^{p+1}},
$$

where $\tau^{\prime}=A \min \left\{h^{2} \varphi_{h m i n}^{-p-1}, \tau\right\}$ and $A=1-2^{N}\|J\|_{\infty}\left\|\varphi_{h}\right\|_{\infty}^{p+1}$. 
Proof. We know from Remark 3.1 that $\left\|U_{h}^{(n)}\right\|_{\infty} \leq\left\|\varphi_{h}\right\|_{\infty}$. Since

$$
\sum_{L \in \Gamma_{*}} h^{N} J\left(x_{K}-x_{L}\right) \leq 2^{N}\|J\|_{\infty}, \quad K \in \Gamma,
$$

exploiting (5), we see that

$$
\delta_{t} U_{K}^{(n)} \leq 2^{N}\|J\|_{\infty}\left\|\varphi_{h}\right\|_{\infty}-\left(U_{K}^{(n)}\right)^{-p}, \quad K \in \Gamma, \quad n \geq 0
$$

or equivalently

$$
\delta_{t} U_{K}^{(n)} \leq-\left(U_{K}^{(n)}\right)^{-p}\left(1-2^{N}\|J\|_{\infty}\left\|\varphi_{h}\right\|_{\infty}\left(U_{K}^{(n)}\right)^{p}\right), \quad K \in \Gamma, \quad n \geq 0 .
$$

Use the fact that $\left\|U_{h}^{(n)}\right\|_{\infty} \leq\left\|\varphi_{h}\right\|_{\infty}, n \geq 0$ to arrive at

$$
\delta_{t} U_{K}^{(n)} \leq-\left(U_{K}^{(n)}\right)^{-p}\left(1-2^{N}\|J\|_{\infty}\left\|\varphi_{h}\right\|_{\infty}^{p+1}\right), \quad K \in \Gamma, \quad n \geq 0 .
$$

These estimates may be rewritten as follows

$$
U_{K}^{(n+1)} \leq U_{K}^{(n)}-A \Delta t_{n}\left(U_{K}^{(n)}\right)^{-p}, \quad K \in \Gamma, \quad n \geq 0 .
$$

Let $K_{0} \in \Gamma$ be such that $U_{K_{0}}^{(n)}=U_{h m i n}^{(n)}$. Replacing $K$ by $K_{0}$ in (16), we note that

$$
U_{K_{0}}^{(n+1)} \leq U_{h m i n}^{(n)}-A \Delta t_{n}\left(U_{h m i n}^{(n)}\right)^{-p}, \quad n \geq 0
$$

which implies that

$$
U_{h m i n}^{(n+1)} \leq U_{h m i n}^{(n)}-A \Delta t_{n}\left(U_{h m i n}^{(n)}\right)^{-p}, \quad n \geq 0,
$$

because $U_{K_{0}}^{(n+1)} \geq U_{h m i n}^{(n+1)}$. We observe that

$$
A \Delta t_{n}\left(U_{h m i n}^{(n)}\right)^{-p-1}=A \min \left\{h^{2}\left(U_{h m i n}^{(n)}\right)^{-p-1}, \tau\right\} .
$$

Exploiting (17), we see that $U_{h m i n}^{(n+1)} \leq U_{h m i n}^{(n)}, n \geq 0$, and by induction, we note that $U_{h m i n}^{(n)} \leq$ $U_{h \min }^{(0)}=\varphi_{\text {hmin }}$. In view of (18), we discover that

$$
A \Delta t_{n}\left(U_{h m i n}^{(n)}\right)^{-p-1} \geq A \min \left\{h^{2} \varphi_{h m i n}^{-p-1}, \tau\right\}=\tau^{\prime} .
$$

Therefore, employing (17), we get

$$
U_{h m i n}^{(n+1)} \leq U_{h m i n}^{(n)}\left(1-\tau^{\prime}\right), \quad n \geq 0 .
$$

Using an argument of recursion, we find that

$$
U_{h m i n}^{(n)} \leq U_{h m i n}^{(0)}\left(1-\tau^{\prime}\right)^{n}=\varphi_{h m i n}\left(1-\tau^{\prime}\right)^{n}, \quad n \geq 0 .
$$


This implies that $U_{h m i n}^{(n)}$ goes to zero as $n$ approaches infinity. Now, let us estimate the discrete quenching time. The restriction on the time step and (21) lead us to

$$
\sum_{n=0}^{\infty} \Delta t_{n} \leq \tau \varphi_{h \min }^{p+1} \sum_{n=0}^{\infty}\left(\left(1-\tau^{\prime}\right)^{p+1}\right)^{n}
$$

Use the fact that the series on the right hand side of the above inequality converges towards $\frac{1}{1-\left(1-\tau^{\prime}\right)^{p+1}}$ to complete the rest of the proof.

Remark 4.1. Due to (20), an argument of recursion reveals that

$$
U_{h \min }^{(n)} \leq U_{h \min }^{(q)}\left(1-\tau^{\prime}\right)^{n-q}, \quad n \geq q .
$$

In view of the above estimates, the restriction on the time step allows us to write

$$
\sum_{n=q}^{\infty} \Delta t_{n} \leq \tau\left(U_{h \min }^{(q)}\right)^{p+1} \sum_{n=q}^{\infty}\left(\left(1-\tau^{\prime}\right)^{p+1}\right)^{n-q} .
$$

Since the series on the right hand side of the above inequality converges towards $\frac{1}{1-\left(1-\tau^{\prime}\right)^{p+1}}$, we infer that $\sum_{n=q}^{\infty} \Delta t_{n} \leq \frac{\tau\left(U_{h m i n}^{(q)}\right)^{p+1}}{1-\left(1-\tau^{\prime}\right)^{p+1}}$, or equivalently

$$
T_{h}^{\Delta t}-t_{q} \leq \frac{\tau\left(U_{h \min }^{(q)}\right)^{p+1}}{1-\left(1-\tau^{\prime}\right)^{p+1}} .
$$

Apply Taylor's expansion to obtain $\left(1-\tau^{\prime}\right)^{p+1}=1-(p+1) \tau^{\prime}+o\left(\tau^{\prime}\right)$. This implies that $\frac{\tau}{1-\left(1-\tau^{\prime}\right)^{p+1}}=$ $\frac{\tau}{\tau^{\prime}((p+1)+o(1))}$. Due to the fact that $\tau^{\prime}=A \min \left\{h^{2} \varphi_{h m i n}^{-p-1}, \tau\right\}$, if we choose $\tau=h^{2}$, then we note that $\frac{\tau^{\prime}}{\tau}=A \min \left\{\varphi_{\text {hmin }}^{-p-1}, 1\right\}$, which implies that $\frac{\tau}{\tau^{\prime}}=O(1)$ with the choice $\tau=h^{2}$.

In the sequel, we pick $\tau=h^{2}$.

Under the assumption of the above theorem, we have seen that the discrete solution quenches in a finite time, and an estimation of its numerical quenching time has been given. In the theorem below, we derive an upper bound of the numerical quenching time taking into account the assumption of the earlier theorem.

Theorem 4.2. Assume that $B h^{2}<1$, where $B=1+2^{N}\|J\|_{\infty}\left\|\varphi_{h}\right\|_{\infty}^{p+1}$. Then, under the hypothesis of Theorem 4.1, the discrete solution $U_{h}^{(n)}$ of (5)-(6) quenches in a finite time, and its quenching time $T_{h}^{\Delta t}$ satisfies the following estimate

$$
T_{h}^{\Delta t} \geq \frac{h^{2} \min \left\{1, \varphi_{h \min }^{p+1}\right\}}{1-\left(1-B h^{2}\right)^{p+1}}
$$

Proof. We know from Theorem 4.1 that the discrete solution quenches in a finite time. Thus, our purpose is to establish the above estimate. Employing (5), we note that

$$
\delta_{t} U_{K}^{(n)} \geq-2^{N}\|J\|_{\infty}\left\|\varphi_{h}\right\|_{\infty}-\left(U_{K}^{(n)}\right)^{-p}, \quad K \in \Gamma, \quad n \geq 0 .
$$


To obtain the above inequalities, we have used the fact that $J$ is nonnegative, $\left\|U_{h}^{(n)}\right\|_{\infty} \leq\|\varphi\|_{\infty}$ and $\sum_{L \in \Gamma_{*}} h^{N} J\left(x_{K}-x_{L}\right) \leq 2^{N}\|J\|_{\infty}, K \in \Gamma$. Since $0 \leq U_{K}^{(n)} \leq\left\|\varphi_{h}\right\|_{\infty}, K \in \Gamma$, the inequalities (22) become

$$
\delta_{t} U_{K}^{(n)} \geq-B\left(U_{K}^{(n)}\right)^{-p}, \quad K \in \Gamma, \quad n \geq 0 .
$$

Taking into account the restriction on the time step, it is not hard to see that

$$
\Delta t_{n}\left(U_{K}^{(n)}\right)^{-p-1} \leq \Delta t_{n}\left(U_{h \min }^{(n)}\right)^{-p-1} \leq h^{2}, \quad K \in \Gamma, \quad n \geq 0 .
$$

In view of (23) and (24), we infer that

$$
U_{K}^{(n+1)} \geq U_{K}^{(n)}\left(1-B h^{2}\right), \quad K \in \Gamma, \quad n \geq 0,
$$

which implies that

$$
U_{h \min }^{(n+1)} \geq U_{h \min }^{(n)}\left(1-B h^{2}\right), \quad n \geq 0 .
$$

By induction, we realize that

$$
U_{h m i n}^{(n)} \geq U_{h \min }^{(0)}\left(1-B h^{2}\right)^{n}=\varphi_{h \min }\left(1-B h^{2}\right)^{n}, \quad n \geq 0 .
$$

Now, let us estimate the numerical quenching time. The restriction on the time step and (26) reveal that

$$
\sum_{n=0}^{\infty} \Delta t_{n} \geq \sum_{n=0}^{\infty} \min \left\{h^{2}, h^{2} \varphi_{h \min }^{p+1}\left(\left(1-B h^{2}\right)^{p+1}\right)^{n}\right\},
$$

which implies that

$$
\sum_{n=0}^{\infty} \Delta t_{n} \geq \min \left\{h^{2}, h^{2} \varphi_{h \min }^{p+1}\right\} \sum_{n=0}^{\infty}\left(\left(1-B h^{2}\right)^{p+1}\right)^{n} .
$$

Use the fact that the series on the right hand side of the above inequality converges towards $\frac{1}{1-\left(1-B h^{2}\right)^{p+1}}$ to complete the rest of the proof.

Remark 4.2. Apply Taylor's expansion to obtain

$$
\left(1-B h^{2}\right)^{p+1}=1-B(p+1) h^{2}+o\left(h^{2}\right),
$$

which implies that $\frac{h^{2}}{1-\left(1-B h^{2}\right)^{p+1}}=\frac{1}{B(p+1)+o(1)}$.

\section{Convergence of the Numerical Quenching Time}

In this section, we denote by

$$
v_{h}(t)=\left(v\left(x_{K}, t\right)\right)_{K \in \Gamma} .
$$

Under some hypotheses, we prove that the discrete solution quenches in a finite time, and its numerical quenching time converges to the real one when the mesh size goes to zero.

We need the following lemma. 
Lemma 5.1. Let $f \in C^{1}(\bar{\Omega})$. Then, we have

$$
\int_{\Omega} f(x) d x=\sum_{J \in \Gamma_{*}} h^{N} f\left(x_{J}\right)+O(h)
$$

The proof of the above lemma is based on the fact that, if $g \in C^{1}([-1,1])$, then $\int_{-1}^{1} g(x) d x=$ $\sum_{j=0}^{I-1} h g\left(x_{j}\right)+O(h)$. Making use of the above result, and exploiting Fubini's theorem, one easily proves the above lemma.

In order to obtain the result concerning the convergence of the numerical quenching time, we firstly prove that the discrete solution approaches the real one in any interval $\bar{\Omega} \times[0, T-\tau]$ with $\tau \in(0, T)$. This result is stated in the following theorem.

Theorem 5.1. Assume that the problem (3)-(4) admits a solution $v \in C^{1,2}(\bar{\Omega} \times[0, T-\tau])$ such that $\min _{t \in[0, T-\tau]} v_{\min }(t)=\alpha>0$ with $\tau \in(0, T)$. Suppose that the initial data at (6) satisfies

$$
\left\|\varphi_{h}-v_{h}(0)\right\|_{\infty}=o(1) \quad \text { as } \quad h \rightarrow 0
$$

Then, the problem (5)-(6) admits a unique solution $U_{h}^{(n)}$ for $h$ small enough, $n \leq R$, and the following relation holds

$$
\sup _{0 \leq n \leq R}\left\|U_{h}^{(n)}-v_{h}\left(t_{n}\right)\right\|_{\infty}=O\left(\left\|\varphi_{h}-v_{h}(0)\right\|_{\infty}+h\right) \quad \text { as } \quad h \rightarrow 0
$$

where $R$ is a positive integer such that $\sum_{j=0}^{R-1} \Delta t_{j} \leq T-\tau$, and $t_{n}=\sum_{j=0}^{n-1} \Delta t_{j}$.

Proof. The problem (5)-(6) admits for each $n \geq 0$, a unique solution $U_{h}^{(n)}$. Let $d \leq R$ be the greatest integer such that

$$
\left\|U_{h}^{(n)}-v_{h}\left(t_{n}\right)\right\|_{\infty}<\frac{\alpha}{2} \quad \text { for } \quad n<d .
$$

Making use of (28), we note that $d \geq 1$ for $h$ small enough. An application of the triangle inequality renders

$$
U_{h \min }^{(n)} \geq v_{h \min }\left(t_{n}\right)-\left\|U_{h}^{(n)}-v_{h}\left(t_{n}\right)\right\|_{\infty} \geq \alpha-\frac{\alpha}{2}=\frac{\alpha}{2} \quad \text { for } \quad n<d .
$$

Exploit Taylor's expansion and use Lemma 5.1 to obtain

$$
\begin{gathered}
\delta_{t} v\left(x_{K}, t_{n}\right)=v_{t}\left(x_{K}, t_{n}\right)+\frac{\Delta t_{n}}{2} v_{t t}\left(x_{K}, \widetilde{t}_{n}\right), \quad K \in \Gamma, \quad n<d, \\
\int_{\Omega} J\left(x_{K}-y\right)\left(v\left(y, t_{n}\right)-v\left(x_{K}, t_{n}\right)\right) d y \\
=\sum_{L \in \Gamma_{*}} h^{N} J\left(x_{K}-x_{L}\right)\left(v\left(x_{L}, t_{n}\right)-v\left(x_{K}, t_{n}\right)\right)+O(h), \quad K \in \Gamma, \quad n<d,
\end{gathered}
$$


which implies that

$$
\begin{aligned}
& \delta_{t} v\left(x_{K}, t_{n}\right)=\sum_{L \in \Gamma_{*}} h^{N} J\left(x_{K}-x_{L}\right)\left(v\left(x_{L}, t_{n}\right)-v\left(x_{K}, t_{n}\right)\right) \\
& -\left(v\left(x_{K}, t_{n}\right)\right)^{-p}+\frac{\Delta t_{n}}{2} v_{t t}\left(x_{K}, \widetilde{t}_{n}\right)+O(h), \quad K \in \Gamma, \quad n<d .
\end{aligned}
$$

Introduce the error $e_{h}^{(n)}$ defined as follows

$$
e_{K}^{(n)}=U_{K}^{(n)}-v\left(x_{K}, t_{n}\right), \quad K \in \Gamma, \quad n<d .
$$

Invoking the mean value theorem, it is easy to see that

$$
\begin{gathered}
\delta_{t} e_{K}^{(n)}=\sum_{L \in \Gamma_{*}} h^{N} J\left(x_{K}-x_{L}\right)\left(e_{L}^{(n)}-e_{K}^{(n)}\right)+p\left(\xi_{K}^{(n)}\right)^{-p-1} e_{K}^{(n)} \\
-\frac{\Delta t_{n}}{2} v_{t t}\left(x_{K}, \widetilde{t_{n}}\right)+O(h), \quad K \in \Gamma, \quad n<d,
\end{gathered}
$$

where $\xi_{K}^{(n)}$ is an intermediate value between $v\left(x_{K}, t_{n}\right)$ and $U_{K}^{(n)}$. We infer that there exists a positive constant $Q$ such that

$$
\begin{gathered}
\delta_{t} e_{K}^{(n)} \leq \sum_{L \in \Gamma_{*}} h^{N} J\left(x_{K}-x_{L}\right)\left(e_{L}^{(n)}-e_{K}^{(n)}\right)+p\left(\xi_{K}^{(n)}\right)^{-p-1} e_{K}^{(n)} \\
+Q h, \quad K \in \Gamma, \quad n<d,
\end{gathered}
$$

because $v \in C^{1,2}, J \in C^{1}\left(\mathbb{R}^{N}\right)$, and $\Delta t_{n}=O\left(h^{2}\right)$. Introduce the vector $Z_{h}^{(n)}$ defined as follows

$$
Z_{K}^{(n)}=e^{(L+1) t_{n}}\left(\left\|\varphi_{h}-v_{h}(0)\right\|_{\infty}+Q h\right), \quad K \in \Gamma, \quad n<d,
$$

where $L=p\left(\frac{\alpha}{2}\right)^{-p-1}$. A straightforward computation reveals that

$$
\begin{gathered}
\delta_{t} Z_{K}^{(n)} \geq \sum_{L \in \Gamma_{*}} h^{N} J\left(x_{K}-x_{L}\right)\left(Z_{L}^{(n)}-Z_{K}^{(n)}\right)+p\left(\xi_{K}^{(n)}\right)^{-p-1} \\
+Q h, \quad K \in \Gamma, \quad n<d \\
Z_{K}^{(0)} \geq e_{K}^{(0)}, \quad K \in \Gamma .
\end{gathered}
$$

We deduce from Lemma 3.2 that

$$
Z_{K}^{(n)} \geq e_{K}^{(n)}, \quad K \in \Gamma, \quad n<d .
$$


In the same way, we also show that

$$
Z_{K}^{(n)} \geq-e_{K}^{(n)}, \quad K \in \Gamma, \quad n<d,
$$

which implies that $\left\|e_{h}^{(n)}\right\|_{\infty} \leq\left\|Z_{h}^{(n)}\right\|_{\infty}, n<d$, or equivalently

$$
\left\|U_{h}^{(n)}-v_{h}\left(t_{n}\right)\right\|_{\infty} \leq e^{(L+1) t_{n}}\left(\left\|\varphi_{h}-v_{h}(0)\right\|_{\infty}+Q h\right), \quad n<d .
$$

Now, let us reveal that $d=R$. To prove this result, we argue by contradiction. Assume that $d<R$. Replacing $n$ by $d$ in (32), and using (29), we discover that

$$
\frac{\alpha}{2} \leq\left\|U_{h}^{(d)}-v_{h}\left(t_{d}\right)\right\|_{\infty} \leq e^{(L+1) T}\left(\left\|\varphi_{h}-v_{h}(0)\right\|_{\infty}+Q h\right) .
$$

Since the term on the right hand side of the second inequality goes to zero as $h$ tends to zero, we deduce that $\frac{\alpha}{2} \leq 0$, which is impossible. Consequently, $d=R$, and the proof is complete.

Now, we are in a position to prove the main result of this section.

Theorem 5.2. Assume that the problem (3)-(4) has a solution $v$ which quenches in a finite time $T$ such that $v \in C^{1,2}(\bar{\Omega} \times[0, T))$. Suppose that the initial data at (6) satisfies the condition (28). Then, under the hypotheses of Theorem 4.1, the solution $U_{h}^{(n)}$ of (5)-(6) quenches in a finite time, and its numerical quenching time $T_{h}^{\Delta t}$ obeys the following relation

$$
\lim _{h \rightarrow 0} T_{h}^{\Delta t}=T .
$$

Proof. Let $0<\varepsilon<T / 2$. In view of Remark 4.1, we know that $\frac{\tau}{1-\left(1-\tau^{\prime}\right)^{p+1}}$ is bounded. Thus, there exists a positive constant $\rho$ such that

$$
\frac{\tau \rho^{p+1}}{1-\left(1-\tau^{\prime}\right)^{p+1}} \leq \frac{\varepsilon}{2}
$$

Since $u$ quenches at the time $T$, there exists a time $T_{0} \in(T-\varepsilon / 2, T)$ such that $0<v_{h m i n}(t)<$ $\frac{\rho}{2} \quad$ for $\quad t \in\left[T_{0}, T\right)$. Let $q$ be a positive integer such that $t_{q}=\sum_{n=0}^{q-1} \Delta t_{n} \in\left[T_{0}, T\right)$. Invoking Theorem 5.1, we know that the problem (5)-(6) admits a unique solution $U_{h}^{(n)}$ such that $\| U_{h}^{(q)}-$ $v_{h}\left(t_{q}\right) \|_{\infty} \leq \frac{\rho}{2}$. An application of the triangle inequality gives

$$
U_{h \min }^{(q)} \leq v_{h \min }\left(t_{q}\right)+\left\|U_{h}^{(q)}-v_{h}\left(t_{q}\right)\right\|_{\infty},
$$

which implies that $U_{h m i n}^{(q)} \leq \frac{\rho}{2}+\frac{\rho}{2}=\rho$. Taking into account Theorem 4.1, we know that the discrete solution $U_{h}^{(n)}$ quenches in a finite time $T_{h}^{\Delta t}$. It follows from Remark 4.1 and (33) that

$$
\left|T_{h}^{\Delta t}-T\right| \leq\left|T_{h}^{\Delta t}-t_{q}\right|+\left|t_{q}-T\right| \leq \frac{\varepsilon}{2}+\frac{\varepsilon}{2}=\varepsilon .
$$

This finishes the proof. 


\section{Numerical Results}

In this section, we give some computational experiments to illustrate the theory given in the previous section. We consider the problem (3)-(4) in the case where $N=1$,

$$
J(x)=\left\{\begin{array}{l}
\frac{15}{16}\left(1-x^{2}\right)^{2} \quad \text { if } \quad|x| \leq 1 \\
0 \quad \text { if } \quad|x|>1
\end{array}\right.
$$

$v_{0}(x)=4+\cos (\pi x)$. We consider the explicit scheme defined in (5)-(6). We also use the following implicit scheme

$$
\begin{aligned}
& \frac{U_{i}^{(n+1)}-U_{i}^{(n)}}{\Delta t_{n}}=\sum_{l=0}^{I-1} h J\left(x_{i}-x_{l}\right)\left(U_{l}^{(n+1)}-U_{i}^{(n+1)}\right) \\
& -\left(U_{i}^{(n)}\right)^{-p-1} U_{i}^{(n+1)}, \quad 0 \leq i \leq I, \quad U_{i}^{(0)}=\varphi_{i}, \quad 0 \leq i \leq I .
\end{aligned}
$$

In both cases, we take $\varphi_{i}=4+\cos \left(\pi x_{i}\right)$. As in the case of the explicit scheme, here, we also choose

$$
\Delta t_{n}=h^{2}\left(U_{h m i n}^{(n)}\right)^{p+1}
$$

Let us again remark that for the above implicit scheme, existence and positivity of the discrete solution are also guaranteed using standard methods (see, for instance [9]).

In the following tables, in rows, we present the numerical quenching times, the numbers of iterations, the CPU times and the orders of the approximations corresponding to meshes of 16, 32, 64,128 . We take for the numerical quenching time $t_{n}=\sum_{j=0}^{n-1} \Delta t_{j}$ which is computed at the first time when

$$
\Delta t_{n}=\left|t_{n+1}-t_{n}\right| \leq 10^{-16} .
$$

The order $(s)$ of the method is computed from

$$
s=\frac{\log \left(\left(T_{4 h}-T_{2 h}\right) /\left(T_{2 h}-T_{h}\right)\right)}{\log (2)} .
$$

\section{Numerical experiments for $p=1$}

Table 1: Numerical quenching times, numbers of iterations, CPU times (seconds) and orders of the approximations obtained with the explicit Euler method

\begin{tabular}{|l|l|l|l|l|}
\hline$I$ & $t_{n}$ & $n$ & CPU time & $s$ \\
\hline 16 & 4.843163 & 1272 & 8 & - \\
\hline 32 & 4.553751 & 4849 & 40 & - \\
\hline 64 & 4.507777 & 18651 & 308 & 2.65 \\
\hline 128 & 4.501537 & 59860 & 3008 & 2.89 \\
\hline
\end{tabular}


Table 2: Numerical quenching times, numbers of iterations, CPU times (seconds) and orders of the approximations obtained with the implicit Euler method

\begin{tabular}{|l|l|l|l|l|}
\hline$I$ & $t_{n}$ & $n$ & CPU time & $s$ \\
\hline 16 & 4.903291 & 1071 & 5.2 & - \\
\hline 32 & 4.563995 & 4097 & 43 & - \\
\hline 64 & 4.509770 & 15674 & 358 & 2.64 \\
\hline 128 & 4.501537 & 59860 & 9360 & 2.71 \\
\hline
\end{tabular}

Remark 6.1. We observe from Tables 1-2 that the numerical quenching time of the discrete solution is approximately equal to 4.5 .

In what follows, we also give some plots to illustrate our analysis. In Figures 1-2, we can appreciate that the discrete solution quenches in a finite time.

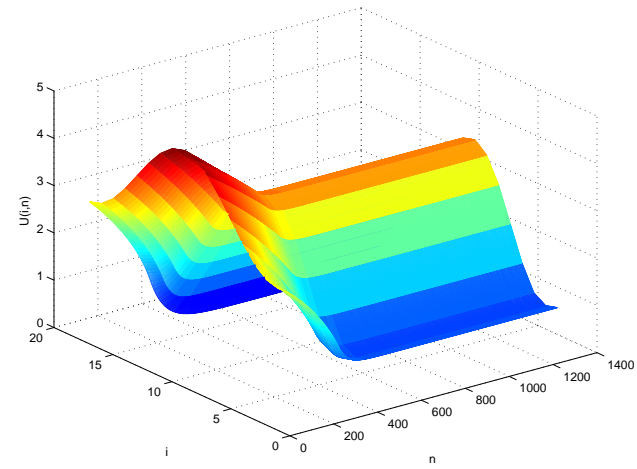

Figure 1: Evolution of the discrete solution (explicit scheme).

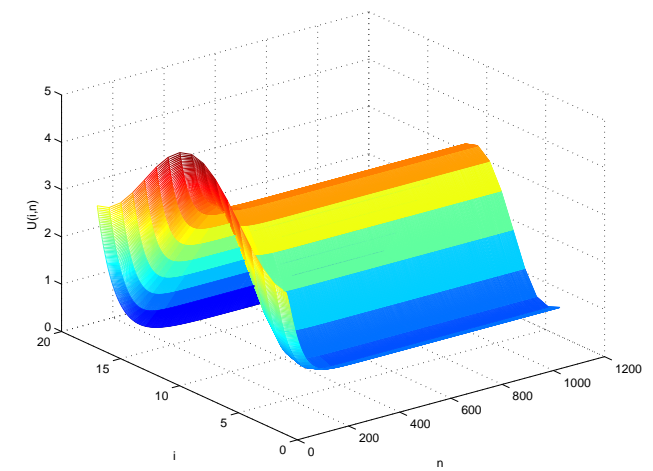

Figure 2: Evolution of the discrete solution (implicit scheme).

Received: October, 2008. Revised: October, 2009.

\section{References}

[1] Andren, F., Mazon, J.M., Rossi, J.D. and Toledo, J., The Neumann problem for nonlocal nonlinear diffusion equations, J. Evol. Equat., 8 (2008), 189-215.

[2] Andren, F., Mazon, J.M., Rossi, J.D. And Toledo, J., A nonlocal p-Laplacian evolution equation with Neumann boundary conditions, Preprint. 
[3] Bates, P. and Chmaj, A., An intergrodifferential model for phase transitions: stationary solutions in higher dimensions, J. Statistical Phys., 95 (1999), 1119-1139.

[4] Bates, P. And Chmaj, A., A discrete convolution model for phase transitions, Arch. Ration. Mech. Anal., 150 (1999), 281-305.

[5] Bates, P. And Han, J., The Dirichlet boundary problem for a nonlocal Cahn-Hilliard equation, J. Math. Anal. Appl., 311 (2005), 289-312.

[6] Bates, P. And Han, J., The Neumann boundary problem for a nonlocal Cahn-Hilliard equation, J. Diff. Equat., 212 (2005), 235-277.

[7] Bates, P., Fife, P. And Wang, X., Travelling waves in a convolution model for phase transitions, Arch. Ration. Mech. Anal., 138 (1997), 105-136.

[8] Boni, T.K., On quenching of solution for some semilinear parabolic equations of second order, Bull. Belg. Math. Soc., 7 (2000), 73-95.

[9] Boni, T.K., Extinction for discretizations of some semilinear parabolic equations, C. R. Acad. Sci. Paris, Sér. I, Math., 333 (2001), 795-800.

[10] Carrilo, C. and Fife, P., Spacial effects in discrete generation population models, J. Math. Bio., 50 (2005), 161-188.

[11] Chasseigne, E., Chaves, M. And Rossi, J.D., Asymptotic behavior for nonlocal diffusion equations whose solutions develop a free boundary, J. Math. Pures et Appl., 86 (2006), $271-291$.

[12] Chen, X., Existence, uniqueness and asymptotic stability of travelling waves in nonlocal evolution equations, Adv. Diff. Equat., 2 (1997), 128-160.

[13] Chen, X.Y. and Matano, H., Convergence, asymptotic periodicity and finite point blow up in one-dimensional semilinear heat equations, J. diff. Equat., 78 (1989), 160-190.

[14] Cortazar, C., Elgueta, M. and Rossi, J.D., A non-local diffusion equation whose solutions develop a free boundary, Ann. Henry Poincaré, 6 (2005), 269-281.

[15] Cortazar, C., Elgueta, M. and Rossi, J.D., How to approximate the heat equation with Neumann boundary conditions by nonlocal diffusion problems, Arch. Ration. Mech. Anal., 187 (2008), 127-156.

[16] Cortazar, C., Elgueta, M., Rossi, J.D. and Wolanski, N., Boundary fluxes for nonlocal diffusion, J. Diff. Equat., 234 (2007), 360-390.

[17] FIfE, P., Some nonclassical trends in parabolic and parabolic-like evolutions. Trends in nonlinear analysis, Springer, Berlin, (2003), 153-191. 
[18] Fife, P. And Wang, X., A convolution model for interfacial motion: the generation and propagation of internal layers in higher space dimensions, Adv. Diff. Equat., 3 (1998), $85-110$.

[19] Friedman, A. And McLeod, B., Blow-up of positive solution of semilinear heat equations, Indiana Univ. Math. J., 34 (1985), 425-447.

[20] Ignat, L.I. and Rossi, J.D., A nonlocal convection-diffusion equation, J. Funct. Anal., 251 (2007), 399-437.

[21] Nabongo, D. And Boni, T.K., Quenching time of solutions for some nonlinear parabolic equations, An. St. Univ. Ovidius Constanta Math., 16 (2008), 87-102.

[22] Nabongo, D. And Boni, T.K., Quenching for semidiscretization of semilinear heat equation with Dirichlet and Neumann boundary conditions, Comment. Math. Univ. Carolinae, 49 (2008), 463-475.

[23] Nabongo, D. And Boni, T.K., Quenching for semidiscretization of a heat equation with singular boundary condition, Asympt. Anal., 59 (2008), 27-38.

[24] Nabongo, D. And Boni, T.K., Blow-up time for a nonlocal diffusion problem with Dirichlet boundary conditions, Comm. Anal. Geom., To appear.

[25] Nabongo, D. And Boni, T.K., Numerical quenching for a semilinear parabolic equation, Math. Modelling and Anal., To appear.

[26] Protter, M.H. and Weinberger, H.F., Maximum principle in differential equations, Prentice Hall, Englewood Cliffs, NJ, (1957)

[27] Perez-Llanos, M. and Rossi, J.D., Blow-up for a non-local diffusion problem with Neumann boundary conditions and a reaction term, Nonl. Anal. TMA, To appear.

[28] Walter, W., Differential-und Integral-Ungleucungen, Springer, Berlin., (1964). 